\section{Ongoing trials in low-grade lymphoma}

\author{
Alexander Burchardt \\ Justus-Liebig University Hospital, Giessen, \\ Germany
}

\begin{abstract}
There are many therapies available for the management of low-grade lymphoma. With follicular lymphoma, for example, combination of chemotherapy and rituximab (immuno-chemotherapy) and consecutive maintenance therapy for 2 years is the current standard of care. To date, the most widely used regimen seems to be rituximab combined with cyclophosphamide, doxorubicin, vincristine, and prednisone ( $\mathrm{R}$ CHOP). Substitution of liposomal doxorubicin in place of conventional doxorubicin may improve outcomes in this indication, although evidence for its use in low-grade lymphoma is not as relevant as in aggressive lymphoma. Bendamustine, in combination with rituximab, has shown very good efficacy and tolerability in several lymphoma types, particularly follicular lymphoma and other low-grade lymphomas. Other combinations, such as those including bortezomib and lenalidomide, are under investigation in low-grade lymphoma, and the duration of rituximab maintenance therapy following bendamustine-rituximab-containing induction is being researched by the German Study Group for Indolent Lymphoma (StiL).
\end{abstract}

\section{Treatment of low-grade lymphoma}

Low-grade lymphomas are chronic diseases, and some patients may live for many years following initial diagnosis. ${ }^{1}$ Therefore, for many patients it is currently regarded as appropriate not to treat immediately at diagnosis, but rather to watch and wait. Once patients do require treatment, there are many therapies available: chemotherapeutic agents known to be effective in low-grade lymphoma include alkylating agents, anthracycline-based regimens, purine analogs, and bendamustine. In addition, there is the established monoclonal antibody therapy rituximab, and newer antibodies such as ofatumumab. Patients may also undergo stem-cell transplantation - either autologous or allogeneic - with or without myeloablation preceding allogeneic transplantation. Finally, there is currently a small amount of experience available on the use of techniques such as DNA vaccination or antisense therapy.

Questions about the long-term outcome of following an initial watchful waiting approach have been raised by a recent study, which compared this approach with immediate treatment with rituximab in patients with stage II, III or IV asymptomatic follicular lymphoma (FL). ${ }^{2}$ The estimated median time to initiation of new therapy among patients in the watchful waiting group was 33 months, whereas among those receiving rituximab the median time was not reached at 4 years $(\mathrm{P}<0.001)$. There were also significant differences in progression-free survival between the observation and rituximab arms $(\mathrm{P}<0.001)$, although no difference in overall survival. These data indicate that initial treatment with rituximab significantly delays the need for new therapy. This may, in the future, change the management approach to patients with newly-diagnosed FL.

It is unclear whether some patients with lowgrade lymphoma can be treated with curative intent, particularly as patients at stages I and II, and some at stage III (for example, those with up to five lymph nodes involved), can live for a long time after diagnosis. Usually treatment is undertaken to manage disease-associated symptoms, such as B symptoms, hematopoietic insufficiency (e.g. anemia, leukopenia, and/or thrombocytopenia), rapid tumor progression, or bulky disease. Some patients develop phenomena such as autoimmune hemolytic anemia or idiopathic thrombocytopenic purpura as a consequence of their disease, and others may have recurrent infections as a result of hypogammaglobulinemia, all of which require treatment.

\section{Standard therapy for low-grade lymphoma}

At this time, combined immuno-chemotherapy with rituximab followed by rituximab maintenance therapy for 2 years is the standard of care for patients with FL (note that the most appropriate maintenance therapy for all other low-grade lymphomas is currently under investigation). What is less clear is the best chemotherapy to combine with rituximab. Chemotherapy combinations comprising cyclophosphamide, doxorubicin, vincristine, and prednisone [CHOP; or cyclophosphamide, vincristine, and prednisone (CVP)] are well accepted by clinicians. In addition, chlorambucil-based chemotherapies such as mitoxantrone, chlorambucil, and prednisone (MCP), and some fludarabine-based therapies [e.g. fludarabine, cyclophosphamide, and mitoxantrone (FCM)] are commonly used, and bendamustine therapy has been investigated with promising results in these patients. Maintenance therapy with rituximab is also the standard of care in patients with low-grade lymphoma. This has been known for some time to be the optimal approach for patients with relapsed disease, and the recent results of the PRIMA study indicate that this is also the most effective treatment strategy for patients with FL after firstline treatment with rituximab plus chemotherapy. ${ }^{3}$
Correspondence: Dr Alexander Burchardt, Department of Hematology, Justus-Liebig University Hospital, Klinikstrasse 33, 35385 Giessen, Germany.

Tel. +49.641.985.42651 - Fax: +49.641.985.42659. E-mail: Alexander.Burchardt@innere.med.unigiessen.de

Key words: low-grade lymphoma, treatment, rituximab, non-pegylated liposomal doxorubicin, bendamustine.

Conflict of interest: the author reports no conflicts of interest.

This work is licensed under a Creative Commons Attribution NonCommercial 3.0 License (CC BYNC 3.0).

(C) Copyright A. Burchardt, 2011

Licensee PAGEPress, Italy

Hematology Reports 2011; 3(s3):e5

doi:10.4081/hr.2011.s3.e5

\section{Liposomal doxorubicin in low-grade lymphoma}

There are currently few data on the use of nonpegylated liposomal doxorubicin (NPLD) specifically in low-grade lymphoma. Some of the publications available are case series, studies involving only small numbers of patients, or studies of patient populations with aggressive as well as low-grade lymphomas. One retrospective study of 37 patients with lymphomas who were either elderly or had cardiac comorbidities included three with FL, two with chronic lymphocytic leukemia, and one with multiple myeloma. 4 These patients received NPLD in combination chemotherapy, and experienced a high rate of remission for this poor-risk population [complete remission rates were $75 \%$ for diffuse large B-cell lymphoma and $55 \%$ for T/NK-cell neoplasm; overall response rates (ORR) of $80 \%$ and $89 \%$, respectively], with no major cardiac toxicities. Rates of hematologic toxicity were comparable to those for regimens containing conventional anthracyclines. ${ }^{4}$ Similarly, in a prospective study of 35 elderly and frail patients, five patients with FL received RCOMP (R-CHOP with NPLD substituted for conventional doxorubicin) as a first-line therapy: $\mathrm{R}$ COMP was effective and well tolerated, and no cardiac events have been observed. ${ }^{5}$ Again, the hematotoxicity of this combination was the same as that seen with the R-CHOP regimen.

A small study has also been conducted using a combination of the new proteasome inhibitor bortezomib with NPLD, fludarabine, and rituximab in 16 patients with refractory/relapsed mantle-cell lymphoma (MCL). ${ }^{6}$ There was a good ORR of $74 \%$ and 9 of 15 patients experienced a complete response (CR) or unconfirmed CR. Rates of neutropenia (37.5\%) and thrombocytopenia (31.2\%) were high, as expected; the rate of cardiotoxicity was $12.5 \%$ (although no information is provided on how this was defined). The authors therefore concluded that this combination therapy was effective and well tolerated. 


\section{Bendamustine in low-grade lymphoma}

The German Study Group for Indolent Lymphoma (StiL) conducted a prospective, randomized Phase III trial (NHL 1-2003) to compare the efficacy of bendamustine plus rituximab (BR) with the widely accepted R-CHOP regimen in patients with low-grade lymphomas. The results were presented at the 2009 American Society of Hematology meeting as an oral presentation.? Bendamustine $\left(90 \mathrm{mg} / \mathrm{m}^{2}\right)$ was administered on days 1 and 2, with rituximab on day 1, every 4 weeks for a maximum of six cycles. R-CHOP was given every 3 weeks, for a maximum of six cycles. In total, 513 patients were evaluable for efficacy and toxicity, most of whom (54\%) had FL, with the remainder having MCL (18\%), marginal zone lymphoma (MZL; 13\%), Waldenström macroglobulinemia (WM; 8\%), and small lymphocytic leukemia (SLL; 4\%). Most patients had stage IV disease (77\%) with bone marrow infiltration.

There was a clear difference between BR and RCHOP in the incidence of grade III and IV hematotoxicities. There were significantly fewer cycles of BR, compared with R-CHOP, in which patients experienced grade III or IV leucocytopenia (12.1\% versus $38.2 \%$, respectively), neutropenia (10.7\% versus $46.5 \%$ ), or required granulocyte colonystimulating factor (4.0 versus $20.0 ; \mathrm{P}<0.0001$ in all cases). BR was associated only with grade 1 alopecia and there was a low incidence of paraesthesias and stomatitis compared with R-CHOP. On the other hand, BR was associated with more skin reactions (erythema) than R-CHOP. Almost all patients in the BR treatment arm received the full dose of chemotherapy ( $96.1 \%$ of cycles), whereas a smaller proportion of cycles of R-CHOP were completed at the full dose of chemotherapy (88.8\%).

The ORR was good in both groups: $93.8 \%$ for BR and $93.5 \%$ for R-CHOP? The CR rate was significantly higher with BR versus R-CHOP (40.1\% versus $30.8 \% ; \mathrm{P}=0.0323$ ). Progression-free survival (PFS) was significantly longer with BR (median PFS 54.8 months) compared with R-CHOP (median 34.8 months) at a median observation period of 32 months [hazard ratio (HR) 0.5765 ; $95 \%$ confidence interval (CI) $0.4292-0.7783 ; \mathrm{P}=0.0002]$. Similarly, event-free survival was 54 months for BR and 31 months for R-CHOP (HR 0.6014; 95\% CI 0.4515-0.7845; $\mathrm{P}=0.0002$ ). Another key measure was the time to next treatment, which had not been reached in the $\mathrm{BR}$ arm versus a median of 40.7 months with R-CHOP (HR 0.5416; 95\% CI 0.3897-0.7491; $\mathrm{P}=0.0002)$. In summary, this study demonstrated that BR significantly improved PFS and $\mathrm{CR}$ rates, compared with R-CHOP, in patients with FL, MCL, and WM. BR was also associated with a better tolerability profile than R-CHOP.

\section{Research into new treatments and strategies for low-grade lymphoma}

The StiL study is now underway to investigate the effect of duration of maintenance ther- apy with rituximab in FL, and the effect of rituximab maintenance therapy in other low-grade lymphoma entities. In the NHL 7-2008 (MAINTAIN) study (NCT00877214), patients with FL will be randomized to either 2 or 4 years of rituximab maintenance therapy, every 2 months, following BR induction therapy. The main efficacy outcome will be PFS. In addition, infectious complications can be studied prospectively in this patient population, which is important because it has been reported that rituximab treatment is associated with neutropenia and increases infectious complications. 8 Another part of the MAINTAIN study is designed to investigate the efficacy of maintenance therapy in other forms of low-grade lymphoma. Patients with WM, MZL, or MCL will be randomized to either 2 years of maintenance therapy with rituximab every 2 months, or 2 years of watch and wait, both following BR induction. Again, efficacy will be measured using PFS.

Studies with other agents are also ongoing. As discussed above, combination therapy with bortezomib, NPLD, fludarabine, and rituximab was shown in a small study to be effective in relapsed MCL. ${ }^{6}$ The StiL NHL 8-2010 study plans to further investigate the efficacy of combination therapy with bortezomib in patients with relapsed low-grade lymphomas. Lenalidomide is another promising therapy in low-grade lymphomas, which has demonstrated good results in patients with several lymphoma types, in hard-to-treat relapsed or refractory disease. 9,10

\section{Conclusions}

Low-grade lymphomas will become chronic diseases, and many patients will require treatment over long periods of time, with many treatment cycles. R-CHOP is commonly used to treat low-grade lymphoma, though chlorambucil- and fludarabine-based regimens are also used. The efficacy and toxicity of R-CHOP may be improved by substitution of NPLD into this combination, although data on the use of liposomal doxorubicin are currently more focused on aggressive lymphomas. BR therapy has produced promising results in several types of lowgrade lymphoma, both in terms of efficacy and safety outcomes, and is recommended in the National Comprehensive Cancer Network (NCCN) Clinical Practice Guidelines in Oncology (V 1.2011) as first-line therapy for indolent lymphoma. ${ }^{11}$ The use of rituximab maintenance treatment after BR induction is currently under investigation in different lymphoma types. In addition, the efficacy of a combination treatment of bortezomib and BR will be studied. The use of lenalidomide also warrants further investigation in low-grade lymphoma.

\section{References}

1. Armitage JO, Weisenburger DD. New approach to classifying non-Hodgkin's lymphomas: clinical features of the major histologic subtypes. Non-Hodgkin's Lymphoma Classification Project. J Clin Oncol 1998;16:2780-95.

2. Ardeshna KM, Qian W, Smith P, et al. An intergroup randomised trial of rituximab versus a watch and wait strategy in patients with stage II, III, IV, asymptomatic, non-bulky follicular lymphoma (grades 1, 2 and 3a). A preliminary analysis. Blood 2010;116:Abs 6.

3. Salles GA, Seymour JF, Feugier P, et al. Rituximab maintenance for 2 years in patients with untreated high tumor burden follicular lymphoma after response to immunochemotherapy. J Clin Oncol 2010;28:15s:Abs 8004.

4. Heintel D, Skrabs C, Hauswirth A, et al. Nonpegylated liposomal doxorubicin is highly active in patients with B and T/NK cell lymphomas with cardiac comorbidity or higher age. Ann Hematol 2010;89:163-9.

5. Gimeno E, Sanchez-Gonzalez B, Alvarez-Larran A, et al. Intermediate dose of nonpegylated liposomal doxorubicin combination (R-CMyOP) as first line chemotherapy for frail elderly patients with aggressive lymphoma. Leuk Res 2011; 35:3058-62.

6. Orciuolo E, Buda G, Pelosini M, Petrini M. Fludarabine, bortezomib, myocet and rituximab chemotherapy in relapsed and refractory mantle cell lymphoma. Br J Haematol 2009;148:81012.

7. Rummel MJ, Niederle N, Maschmeyer G, et al. Bendamustine plus rituximab is superior in respect of progression free survival and $\mathrm{CR}$ rate when compared to CHOP plus rituximab as first-line treatment of patients with advanced follicular, indolent and mantle-cell lymphomas: final results of a randomized Phase III study of the StiL (Study Group Indolent Lymphomas, Germany). Blood 2009;114:Abs 405.

8. Aksoy S, Dizdar O, Hayran M, Harputluoglu H. Infectious complications of rituximab in patients with lymphoma during maintenance therapy: a systematic review and meta-analysis. Leuk Lymphoma 2009;50:357-65.

9. Witzig TE, Wiernik PH, Moore T, et al. Lenalidomide oral monotherapy produces durable responses in relapsed or refractory indolent non-Hodgkin's lymphoma. J Clin Oncol 2009;27:5404-9.

10. Habermann TM, Lossos IS, Justice G, et al. Lenalidomide oral monotherapy produces a high response rate in patients with relapsed or refractory mantle cell lymphoma. Br J Haematol 2009;145:344-9.

11. National Comprehensive Cancer Network. NCCN Clinical Practice Guidelines in Oncology (NCCN Guidelines ${ }^{\mathrm{TM}}$ ). http//www.nccn.org/ professionals/physician_gls/f_guidelines.asp. 